Check for updates

Cite this: Phys. Chem. Chem. Phys., 2019, 21, 8054

Received 10th December 2018, Accepted 25th March 2019

DOI: $10.1039 / c 8 c p 07544 a$

rsc.li/pccp

\section{$A b$ initio prediction of structuring/mesoscale inhomogeneities in surfactant-free microemulsions and hydrogen-bonding-free microemulsions $\dagger$}

\author{
Maximilian Hahn, ab Sebastian Krickl, ${ }^{\mathrm{b}}$ Thomas Buchecker, ${ }^{\mathrm{c}}$ Gašper Jošt, ${ }^{\mathrm{c}}$ \\ Didier Touraud, ${ }^{b}$ Pierre Bauduin, (D) ${ }^{d}$ Arno Pfitzner, (ID ${ }^{c}$ Andreas Klamt*ab and \\ Werner Kunz iD *b
}

\begin{abstract}
In this paper, we consider the influence of $\mathrm{H}$-bond donor and acceptor functionalities on the formation of mesoscale inhomogeneities in ternary systems. It was found that hydrogen-bonding re-enforces such structures, but is not necessarily a prerequisite for the occurrence of mesoscale, microemulsion-like structuring in ternary surfactant-free microemulsions (SFME) and consequently, hydrogen-bonding-free microemulsions (HBFME) exist. The evaluated ternary systems were investigated by means of dynamic light scattering (DLS) and computer-based calculation methods. Theoretical COSMO-RS based calculations were applied to provide an explanation for different hydrotropic efficiencies, and COSMOplex calculations were used to predict and evaluate the propensity of the molecules to form mesoscale structures in SFME and HBFME. Microemulsion-like fluctuations could be observed in the COSMOplex simulations and correlate fairly well with the appearance of mesoscopic structures observed in SFME and HBFME, although the free energy differences in the formation of aggregate structures in the investigated systems are very small, in the range of $0.05 \mathrm{kcal} \mathrm{mol}^{-1}$.
\end{abstract}

\section{Introduction}

Surfactant-free microemulsions (SFME) are organized ternary solvents consisting of a relatively short amphiphilic component (hydrotrope) and two nearly immiscible liquids, with both of them being to a sufficient extent miscible with the hydrotrope.$^{1-4}$ For a given water/oil system, it can be observed that the liquid-liquid-equilibrium (LLE) miscibility gap gets more and more narrow upon the addition of hydrotrope molecules, until it completely vanishes. The particular minimum molar fraction of hydrotrope, $x_{\min }$ (hydrotrope), at which exclusively monophasic mixtures are obtained independently of the residual molar ratio of water/oil can be considered as a measure for the hydrotropic efficiency of a hydrotrope to co-solubilise water and oil. Mesoscopic organization of ternary water/hydrotrope/oil

\footnotetext{
${ }^{a}$ COSMOlogic GmbH \& Co. KG, Imbacher Weg 46, 51379 Leverkusen, Germany. E-mail:klamt@cosmologic.de

${ }^{b}$ Institute of Physical and Theoretical Chemistry, University of Regensburg, 93040 Regensburg, Germany. E-mail:werner.kunz@chemie.uni-regensburg.de ${ }^{c}$ Institute of Inorganic Chemistry, University of Regensburg, 93040 Regensburg, Germany

${ }^{d}$ Institut de Chimie Séparative de Marcoule (ICSM), UMR 5257 (CEA, CNRS, UM, ENSCM), BP 17171, 30207 Bagnols-sur-Cèze, France

$\dagger$ Electronic supplementary information (ESI) available. See DOI: 10.1039/c8cp07544a
}

systems into oil-rich and water-rich domains is comparable to mesoscopic organization observed in water/surfactant/oil ternary systems (direct, bicontinuous and reverse microemulsions), as previously shown by means of scattering techniques, molecular dynamics simulations and conductivity measurements ${ }^{5-10}$

The unique mesoscopic structuring observed in SFME in the monophasic region of a ternary phase diagram close to the miscibility gap - also known as the pre-ouzo region ${ }^{11}$ - offers great opportunities: SFME are e.g. powerful solubilisation media, ${ }^{2,12}$ provide anomalies in enzymatic activity ${ }^{13,14}$ and chemical reactivity when used as reaction media ${ }^{14-16}$ or can serve as nano-reactors for the templated synthesis of nanoparticles. Other industrial applications can be found in literature. ${ }^{17,18}$

In order to provide a general explanation for the formation and thermodynamic stability of SFME, Zemb et al. recently developed an extended Derjaguin-Landau-Verwey-Overbeek (DLVO) like theory, describing the subtle balance between free energy of hydration (hydration force between surfaces), van-der-Waals interactions (attractive dispersion forces) and mixing entropy (homogeneous mixing of three components), leading to a minimum of the total free energy of SFME, similar (although less pronounced) to conventional microemulsions and micellar solutions. ${ }^{4}$ Other promising approaches to describe hydrotropic solubilisation by use of statistical thermodynamics, 
as e.g., Kirkwood Buff theory, can be found in contributions of Abbott et $a .^{19}$ and Shimizu et al. ${ }^{20-23}$ Nonetheless, there is still a lack of studies that systematically investigate the formation of SFME based on the molecular structures and the corresponding polarity distributions on the molecular surface of the SFME constituents in order to provide more application-orientated rules to formulate SFME and to predict their formation.

In this context, the present contribution focuses on the computer-based prediction of structuring and aggregation in SFME with the COSMO-RS ${ }^{24-26}$ (Conductor-like Screening Model for Realistic Solvation) based COSMOplex method ${ }^{27}$ and evaluates the effects of short hydrotropic components like ethanol, acetone and tetrahydrofuran (THF) on the formation of mesoscale inhomogeneities in various ternary mixtures. To this purpose water/hydrotrope/anisole and water/hydrotrope/ limonene systems were evaluated. Beside of these SFME systems, we focused especially on ternary systems without any hydrogenbonding between the molecules. For simplicity and in analogy to SFME, we will call the observed systems hydrogen-bonding-free microemulsions (HBFME).

The following questions are essential in this context:

- What is the general role of hydrogen bonding in the formation of surfactant-free microemulsions? Note that the role of hydrogen bonding in the formation of SFME has been already questioned by Zemb et al. ${ }^{4}$

- Can predictive, computer-based calculation methods like COSMO-RS or COSMOplex provide an insight into the rather complex topic of self-aggregation or even predict structuring in a self-consistent $a b$ initio calculation?

- Is the occurrence of mesoscale inhomogeneities related with the LLE miscibility gap and is microemulsion-like structuring dependent on the proximity to this miscibility gap, or more particular to the LLE critical point in the ternary phase diagram?

- Can the observed structuring in the pre-Ouzo regions in ternary phase diagrams with different hydrotropic components be correlated with their hydrotropic efficiencies?

In order to address all these questions, we investigated the formation of microemulsions and mesoscale inhomogeneities in ternary water/hydrotrope/anisole, water/hydrotrope/limonene and acetonitrile/hydrotrope/limonene systems with ethanol, acetone and THF as hydrotropes. Typical structures of the used molecules are visualized in Fig. 1 with underlying molecularshaped COSMO surface cavities to indicate the polarities of the molecules. Note that within this study, all COSMO surfaces are color coded by polarization charge densities (also referred to as screening charge densities or $\sigma$-values): deep red and deep blue COSMO surface areas indicate highly positive (red) and negative (blue) screening charge densities on the surface of the molecular-shaped cavity and hence highly negative and positive parts of underlying molecular surface, respectively. These surfaces are highly polar and potentially hydrogen-bonding. Non-polar (neutral) areas are shown in green.

Ternary phase diagrams were recorded and predicted with COSMOtherm $^{28,29}$ to determine the monophasic regions, where SFME formation may occur. COSMOplex ${ }^{27}$ calculations were (a)

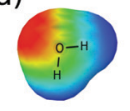

(e)

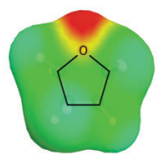

(b)

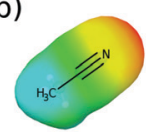

(f)

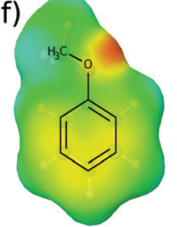

(c)

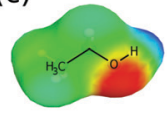

(d)

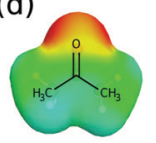

(g)

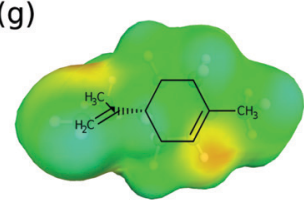

Fig. 1 Chemical structures with underlying COSMO surface cavities to indicate the polarities of the molecules: (a) water, (b) acetonitrile, (c) ethanol, (d) acetone, (e) THF, (f) anisole and (g) limonene. COSMO surfaces, color coded by polarization charge densities: Deep red and deep blue COSMO surface areas indicate highly positive (red) and negative (blue) screening charge densities on the surface of the molecular-shaped cavity and hence highly negative and positive parts of underlying molecular surface, respectively. Non-polar (neutral) areas are shown in green.

applied to predict self-organization and microemulsion-like structuring in the inhomogeneous SFME and HBFME systems and to evaluate the affinity of the molecules to aggregate. Dynamic light scattering (DLS) was used to detect and quantify the size of mesoscale inhomogeneities in the monophasic regions of the different ternary systems. Based on the experimental and theoretical results, conclusions were drawn to provide explanations for the questions asked in the introduction.

\section{Methods and techniques}

\section{COSMO-RS based calculations}

For the theoretical investigation of the recorded phase diagrams, COSMO-RS based calculations were performed using COSMOtherm $^{28,29}$ (version 18.0.0). The Conductor-like Screening Model for Realistic Solvation (COSMO-RS ${ }^{24-26}$ ), combines quantum chemical DFT-BP86 ${ }^{30,31} / \mathrm{COSMO}^{32}$ calculations with statistical thermodynamics and allows for the prediction of a broad range of physico-chemical properties of molecules in solution with reasonable accuracy. Basically, the COSMO-RS model represents an extension of the continuum solvation model COSMO ${ }^{32}$ in which the chemical environment of a solute molecule (the solvent surrounding a molecule of interest) is approximated as a homogeneously polarizable dielectric continuum, whereby the polarization charge densities of the dielectric medium (solvent) are approximated by the screening charges of a virtual conductor. The COSMO polarization charge densities presented as a $\sigma$-surface (also known as COSMO surface: solute-continuum interface as shown in Fig. 1) or $\sigma$-profile (histogram of charged surface segments of a molecule) are extremely useful for the quantification of interactions in solution or the qualitative identification of molecular properties, like e.g. polarity.

In its simplest form, the COSMO-RS model makes use of the new reference state of perfectly screened molecules in a conductor with infinite dielectric and assumes that all relevant interactions between molecules in solution can be expressed as 
local contact energies between COSMO surface segments. Consequently, a dense liquid system is considered as an ensemble of pairwise interacting surface segments and all thermodynamic properties of interest can be calculated from the statistical thermodynamics of this ensemble of interacting surface pieces. The chemical potentials of a segment with polarization charge density $\sigma$ in such an ensemble (the so-called $\sigma$-potential) must be solved iteratively and is exactly described by

$$
\mu_{\mathrm{S}}(\sigma)=-R T \ln \sum_{\sigma^{\prime}} \exp \left[\frac{E\left(\sigma, \sigma^{\prime}\right)-\mu_{\mathrm{S}}\left(\sigma^{\prime}\right)}{R T}\right]
$$

where $E\left(\sigma, \sigma^{\prime}\right)$ denotes the interaction energy between the segments $\sigma$ and $\sigma^{\prime}$ and the sum runs over all segments in the system. The chemical potential of a component $\mathrm{X}$ in a system $\mathrm{S}$ can be calculated from the probability weighted chemical potentials of the individual COSMO surface segments and a combinatorial contribution, $\left(\mu_{\mathrm{C}, \mathrm{S}}^{\mathrm{X}}\right)$ accounting for size and shape differences of the molecules in the system:

$$
\mu_{\mathrm{S}}^{\mathrm{X}}=\sum_{\sigma} p^{\mathrm{X}}(\sigma) \mu_{\mathrm{S}}(\sigma)+\mu_{\mathrm{C}, \mathrm{S}}^{\mathrm{X}}
$$

All thermodynamic equilibrium constants (like e.g. the activity coefficients, partition coefficients or phase compositions in a liquid-liquid phase equilibrium) can be calculated as differences of such chemical potentials as given in Table 1.

Within this study, liquid-liquid phase equilibria (LLE) calculations for the evaluated ternary systems were performed on FINE $^{25}$ level with a TZVPD ${ }^{30}$ basis set. The LLE results and other calculated data on $\sigma$-profiles, chemical potentials, infinite dilution activity coefficients and partition coefficients for the considered mixture systems are given in the ESI. $\dagger$ For detailed information about COSMO-RS and the mentioned calculation methods, we refer to additional literature. ${ }^{24-26,33}$

In order to extend the self-consistent simulation and property prediction abilities of COSMO-RS towards the self-organizing, inhomogeneous SFME and HBFME systems, the recently developed COSMOplex ${ }^{27}$ method can be used. Within the COSMOplex model, a ternary mixture is considered as a layered liquid in a three-dimensional simulation box, whereby all layers of this simulation box can significantly differ from each other in the layer compositions of the molecules in the system. Furthermore, each COSMOplex simulation box layer is considered as homogeneous liquid and can be described by COSMO-RS.
Basically, COSMOplex makes use of the polarity distribution on the molecular surfaces of the compounds in order to provide a reasonable guess for the overall composition and orientation of all conformers of all molecules in the layers of a simulation box and calculates for each simulation box layer the $\sigma$-potentials of all surface pieces of the molecules, i.e. of all COSMO surface segments, within the particular COSMOplex simulation box layer. In the next step, chemical potentials are calculated for all compounds by systematic sampling over all relevant conformations, center positions and orientations relative to the $z$-axis in the layered simulation box.

For each molecule, conformation, center position and orientation, i.e. for each state of a molecule, the state specific free energy is calculated by the summation of the local $\sigma$-potentials of the COSMO surface segments at their position according to the state under consideration. From all the state specific free energies, the partition function of the compound between the layers of the COSMOplex simulation box, and the full information about the distribution of each molecule in the system can be derived. From the distribution of all molecules in the COSMOplex simulation box, the volume population of each simulation box layer can be calculated, resulting in a pressure function as a response to overpopulation. Taking the pressure into account in the next iteration, the distribution of the molecules with respect to position, orientation and conformations can be iterated to self-consistency.

As shown by Klamt et al, the COSMOplex ${ }^{27}$ method is a powerful and efficient extension of the COSMO-RS model and by iteration of the previously described steps to self-consistency allows for the self-consistent prediction of partition functions, free energies and free energy related properties in various self-organizing inhomogeneous systems like e.g. micelles, microemulsions, liquid-liquid or solid-liquid interfaces or multiphases. In most cases, COSMOplex calculations are based on simulation boxes with a water-rich bulk phase, followed by one or more self-organizing system (s.o.s.) phases containing surfactant molecules, followed by an oil-rich bulk phase.

Within this contribution, the directional version of the COSMOplex code (version 18.07.37) was applied for the first time to SFME and HBFME. In contrast to the concentration gradient in classical COSMOplex calculations, our calculations are based on a lamellar self-organizing geometry and started with layer compositions matching exactly the corresponding overall phase compositions of the molecules in the system.

Table 1 Equilibrium properties and corresponding equations and phase definitions

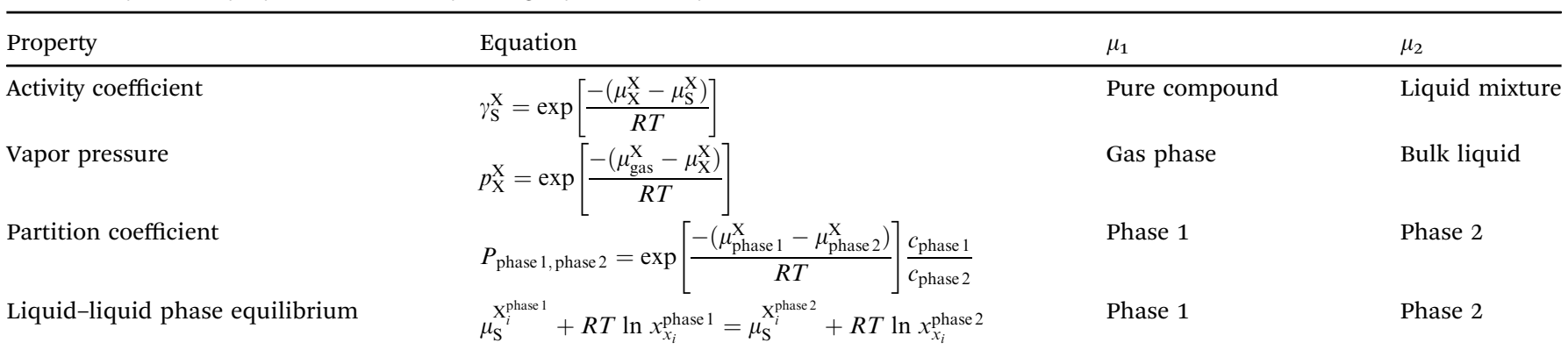


Consequently, all performed calculations can exactly be assigned to points in ternary phase diagrams and therefore be used to predict and evaluate structuring and mesoscale inhomogeneities in SFME and HBFME systems.

Layered simulation boxes of 10 and $20 \mathrm{~nm}$, with 10 layers per $\mathrm{nm}$ of simulation box length, periodic boundary conditions and self-consistent electrostatic potentials were used in all COSMOplex calculations. The simulation temperature was set to $25{ }^{\circ} \mathrm{C}$. Due to the fact that the free energy differences in the formation of mesoscopic structuring in the investigated systems are very small (in the range of $0.05 \mathrm{kcal} \mathrm{mol}^{-1}$ ), we decided not to use the COSMOplex default convergence criteria but applied refined convergence thresholds, which are 100 or 1000 times lower than the default values. In order to ensure the stability of the predicted fluctuations, we recommend that at least the following convergence thresholds should be applied (100 times lower than default values): $\operatorname{rmsd}($ potential profiles) and $\operatorname{rmsd}($ compound free energies $)=0.0001 \mathrm{kcal} \mathrm{mol}^{-1}$ and $\operatorname{rmsd}($ pressure profile $)=$ 0.01 bar.

Several different calculation set-ups have been evaluated (see also ESI $\dagger$ ), differing by the initial composition of the phases in the simulation box, the orientation of the compounds within the phases (with polarity moments ${ }^{34}$ of the compounds parallel to the $z$-axis of the simulation box indicated by arrows in our notation):

- Mixture bulk phase (9 nm), s.o.s. phase (1 nm), s.o.s. phase $(1 \mathrm{~nm})$, mixture bulk phase $(9 \mathrm{~nm})$ - with symmetric polarity moments of the molecules in the s.o.s. phases: (bulk $\leftarrow \rightarrow$ bulk) or (bulk $\rightarrow \leftarrow$ bulk)

- Mixture bulk phase (3.5 nm), 6 s.o.s. phases (0.5 nm each) with alternating polarity moments, mixture bulk phase (3.5 nm): (bulk $\leftarrow \rightarrow \leftarrow \rightarrow \leftarrow \rightarrow$ bulk)

- 8 s.o.s. phases (1.25 $\mathrm{nm}$ or $2.5 \mathrm{~nm}$ each) - with alternating polarity moments of the molecules in the s.o.s. phases $(\leftarrow \rightarrow$ $\leftarrow \rightarrow \leftarrow \rightarrow \leftarrow \rightarrow)$

- 2 s.o.s. phases (10 nm each) - with alternating polarity moments of the molecules in the s.o.s. phases $(\leftarrow \rightarrow)$

\section{Experimental}

\section{Chemicals}

Ethanol $(\geq 99.8 \%)$ and $(R)-(+)$-limonene (97\%, ee: $98 \%)$ were obtained from Sigma-Aldrich (Steinheim, Germany). Anisole ( $\geq 99 \%$ ) and acetonitrile ( $\geq 99.5 \%$ ) were purchased from Merck (Darmstadt, Germany). Tetrahydrofuran (THF, $\geq 99.5 \%$ ) was purchased from Fischer Scientific (Loughborough, UK) and acetone (100\%) from VWR Chemicals (Fontenay-sois-Bois, France). All chemicals were used without further purification. Aqueous solutions were prepared using deionized water with a resistivity of $18 \mathrm{M} \Omega \mathrm{cm}$.

\section{Ternary phase diagrams}

Phase diagrams were recorded using a dynamic and static process according to Dekker et al. ${ }^{35}$ For this purpose, binary mixtures (each $3 \mathrm{~g}$ ) were prepared in screw-able tubes of borosilicate glass. The third component was added gradually until a visible change in the phase behavior occurred. Measurements were carried out at $25{ }^{\circ} \mathrm{C}$ and phase transition was determined by visual inspection of the samples. Mole fractions and weight fractions were calculated from the mass of the individual components derived from precise weight measurements.

\section{Dynamic light scattering}

Dynamic light scattering (DLS) experiments were performed using a temperature controlled CGS-3 goniometer system from ALV (Langen, Germany) equipped with an ALV-7004/FAST Multiple Tau digital correlator and a vertical-polarized $22 \mathrm{~mW}$ HeNe laser (wavelength $\lambda=632.8 \mathrm{~nm}$ ). Prior to the measurements, all samples were filtered into dust-free cylindrical light scattering cells $(10 \mathrm{~mm}$ outer diameter) using a $0.2 \mu \mathrm{m}$ PTFE membrane filter. The sealed measurement cells could be directly placed into the measurement apparatus. Measurements were performed at a scattering angle $\Theta$ of $90^{\circ}$ after thermostating to $25 \pm 0.1{ }^{\circ} \mathrm{C}$. Data points were collected for $300 \mathrm{~s}$.

Mesoscale structures in SFME are usually highly fluctuating and of no well-defined shape. Thus, normalized intensity autocorrelation functions $g^{(2)}(\tau)-1$ were evaluated qualitatively with regard to their $y$-intercept and decay time $\tau$. As a rule of thumb, it was assumed that a higher intercept of the correlation function for small decay times and larger decay times of the correlation function represent the more time-stable and more pronounced structuring of the SFME.

\section{Results \& discussion}

\section{Mesoscale structuring in SFME and HBFME systems - predictable with COSMOplex?}

Pronounced structuring was predicted in acetonitrile/acetone/ limonene at phase compositions of $0.30 / 0.35 / 0.35$ and $0.50 /$ $0.10 / 0.40$ in mole fractions by means of COSMOplex TZVPDFINE level calculations at a temperature of $25{ }^{\circ} \mathrm{C}$ (see Fig. 2) and confirmed by DLS measurements at the same phase compositions (see Fig. 3, left-hand side). At this point we strongly recommend to apply TZVPD-FINE level COSMOplex calculations instead of the default TZVP level COSMOplex calculations for SFME - and especially for HBFME - systems. This is due to a better definition of hydrogen-bonding and some other effects in calculations with TZVPD-FINE level (described and visualized in more detail in the $\mathrm{ESI} \dagger$ ).

Experimental results for the ternary phase diagram strongly deviate from the COSMO-RS predictions (see Fig. 3, right-hand side) and indicate a much lower miscibility gap at lower hydrotrope concentrations. The phase compositions of 0.30 / $0.35 / 0.35$ and $0.50 / 0.10 / 0.40$ in mole fractions corresponds to points in the monophasic region of the ternary phase diagram in proximity to the COSMO-RS-predicted and experimentally measured LLE miscibility gap, respectively. DLS measurements at both phase compositions indicate an even more pronounced structuring at the second point $(0.50 / 0.10 / 0.40)$. 

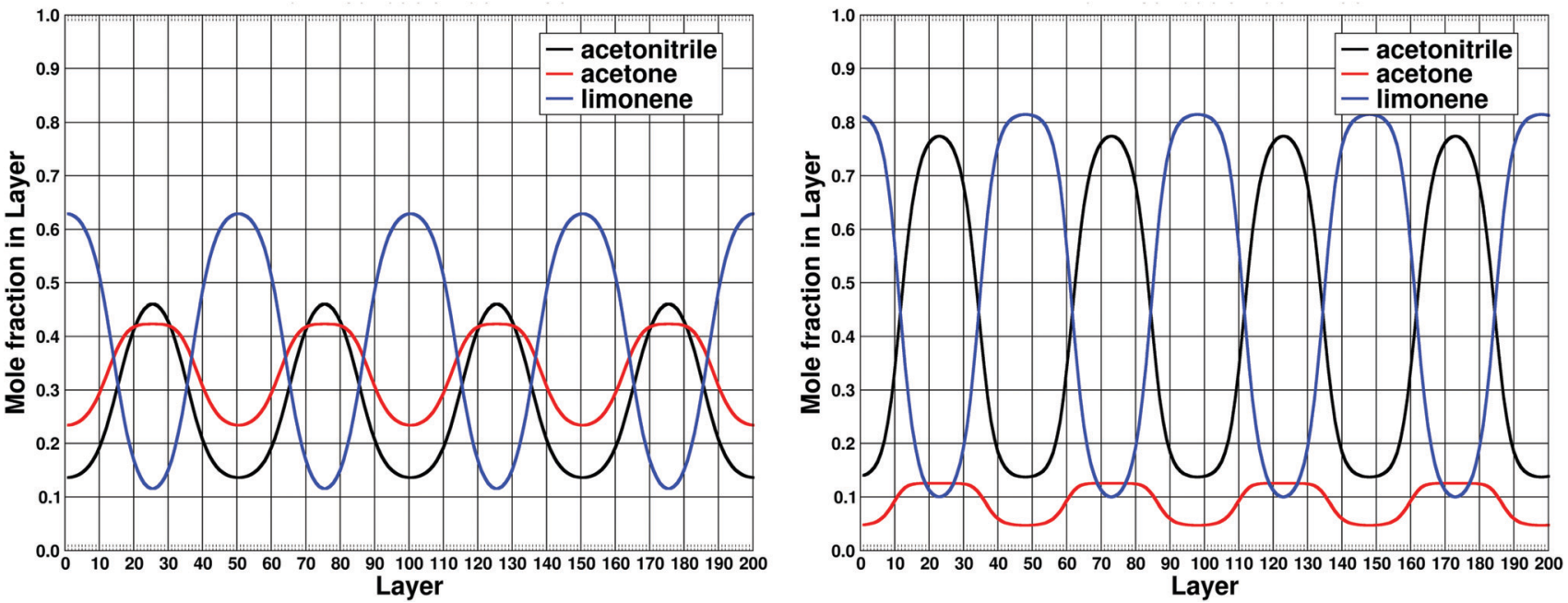

Fig. 2 COSMOplex-predicted fluctuations in acetonitrile/acetone/limonene at phase compositions of 0.30/0.35/0.35 (left) and 0.50/0.10/0.40 (right) in mole fractions: calculations performed on TZVPD-FINE level at a temperature of $25^{\circ} \mathrm{C}$ with 8 s.o.s. phases and alternating polarity moments. Refined convergence thresholds - being 1000 times lower than the COSMOplex default values - were applied. Corresponding DLS results and the phase diagram of the system are compiled in Fig. 3.
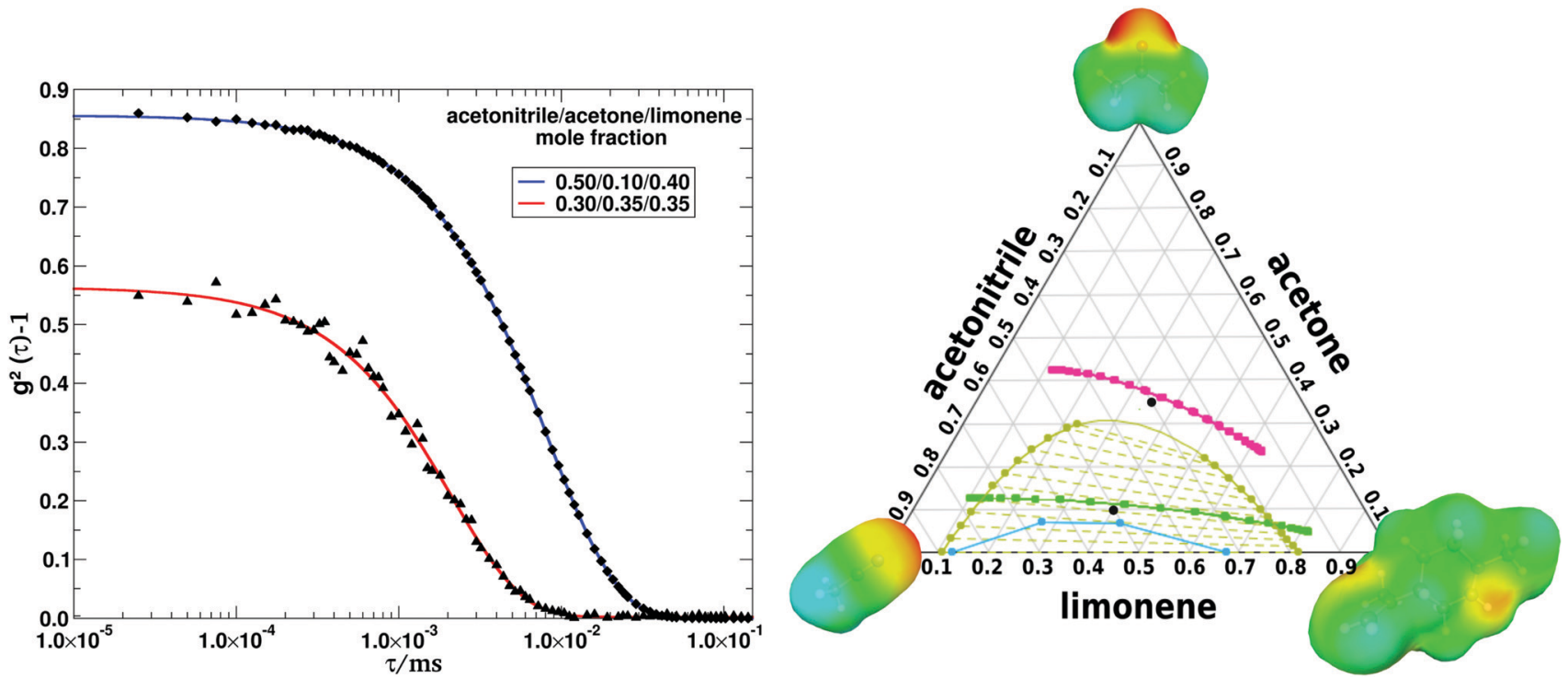

Fig. 3 Correlation functions obtained from DLS experiments (left), measured at mole fraction phase compositions of 0.30/0.35/0.35 and 0.50/0.10/ 0.40 , and ternary phase diagram (light blue: experiment, flat green: predicted with COSMO-RS) of acetonitrile/acetone/limonene (right) - including COSMOplex-predicted fluctuations around the overall mole fraction phase compositions, which are indicated by the black dots in the ternary phase diagram. COSMO-RS and COSMOplex calculations were performed on TZVPD-FINE level at a temperature of $25^{\circ} \mathrm{C}$. 8 s.o.s. phases and alternating polarity moments were used for the COSMOplex calculation set-up and convergence thresholds - being 100 times lower than the COSMOplex default values - were applied.

The existence of mesoscale inhomogeneities and structuring in the described HBFME system is an extraordinary finding which was not reported in literature, to the best of our knowledge - and helps answering the question about the general role of hydrogen bonding on the formation of structured surfactant-free microemulsions.

For the evaluated SFME and HBFME systems, we found an interesting correlation between relative amplitude heights of COSMOplex-predicted, periodic fluctuations (relative means divided by the corresponding mole fraction of the compound in the simulation box), the corresponding path lengths of the fluctuations when plotted in the ternary phase diagram (see right-hand side of Fig. 3), and the propensity of the molecules to form mesoscale structures. Calculated as free energy differences of COSMOplex calculations in homogeneous and inhomogeneous simulation boxes, compound and system free energies of aggregation and self-organization and the corresponding free energy fluctuations in the inhomogeneous 
simulation box - which are due to the inhomogeneities in the layer compositions of the molecules in the COSMOplex simulation box layers - can be used as additional measures for the propensity of the molecules to form mesoscale structures.

Mean values for the compound and system free energies of aggregation and self-organization of the molecules in acetonitrile/acetone/limonene at phase compositions of $0.30 / 0.35$ / 0.35 and $0.50 / 0.10 / 0.40$ in mole fractions and rmsd values of the amplitude heights of the corresponding COSMOplexpredicted free energy fluctuations in the inhomogeneous simulation box are compiled in Table 2 . Note that the amplitude heights of the COSMOplex-predicted free energy fluctuations (see last column of Table 2) are in good correlation with the corresponding relative amplitude heights of the COSMOplexpredicted fluctuations shown in Fig. 2. Furthermore, results indicate that the aggregation of limonene molecules can be seen as the driving force for the observed microemulsion-like structuring in the HBFME system acetonitrile/acetone/limonene.

Thus, COSMOplex calculations provide valuable insights into the rather complex topic of self-organization and results from COSMOplex calculations at different phase compositions can be compared with each other in order to identify and evaluate in which regions of the phase diagram pronounced structuring is rather likely.
Results obtained with two distinct sets of convergence thresholds - being 100 and 1000 times lower than the COSMOplex default values - indicate a strong dependence of the predicted fluctuation amplitude heights and free energies of aggregation and self-organization on the applied convergence thresholds. Be aware that stricter convergence criteria correspond to a higher number of iterations in the self-consistent COSMOplex calculations. Hence, for simulations differing only in the applied convergence thresholds (see Fig. 4 and figures in the ESI $\dagger$ ), the relative differences in the fluctuation amplitude heights and system free energies of aggregation and self-organization can be interpreted as a system, composition and temperature dependent simulation gradient or energy gradient, respectively. These gradients can be seen as a measure for the stability of the predicted fluctuations and the reliability of the COSMOplex predictions with the applied convergence criteria itself.

For phase compositions far from the LLE miscibility gap in ternary phase diagrams, a fast decay of the predicted fluctuation amplitude heights with increasing number of COSMOplex iterations (due to stricter convergence criteria) was observed (see figures in the $\mathrm{ESI}_{\dagger} \dagger$ ). The corresponding large negative simulation gradient indicates that the predicted fluctuations are relatively unstable and will further decay and

Table 2 Mean compound and system free energies of aggregation and self-organization in acetonitrile/acetone/limonene at $25^{\circ} \mathrm{C}$. The mean system free energies $\Delta G_{\text {s.o.s. }}^{0}$ and $\Delta G_{\text {s.o.s. }}$ are mole fraction weighted sums of the mean compound free energy differences of aggregation and self-organization with and without contributions arising from the pressure function and the electrostatic potential, respectively. The amplitude heights of free energy fluctuations $\left(h_{\text {fluct. }}\left(\Delta G_{\text {s.o.s. }}\right)\right)$ corresponding to $\Delta G_{\text {s.o.s. }}$ are indicated by their rmsd values in the last column. All energy values are given in the unit kcal $\mathrm{mol}^{-1}$ and correspond to COSMOplex calculations on TZVPD-FINE level with $(\leftarrow \rightarrow \leftarrow \rightarrow \leftarrow \rightarrow \leftarrow \rightarrow$ ) calculation set-up and refined convergence thresholds (100 times lower than the COSMOplex default values)

\begin{tabular}{|c|c|c|c|c|c|c|}
\hline Phase composition & \multicolumn{3}{|c|}{ Compound free energy differences } & \multicolumn{3}{|c|}{ System free energy differences } \\
\hline $0.30 / 0.35 / 0.35$ & 0.09073 & 0.03537 & -0.22938 & -0.04068 & -0.04112 & \pm 0.12375 \\
\hline
\end{tabular}
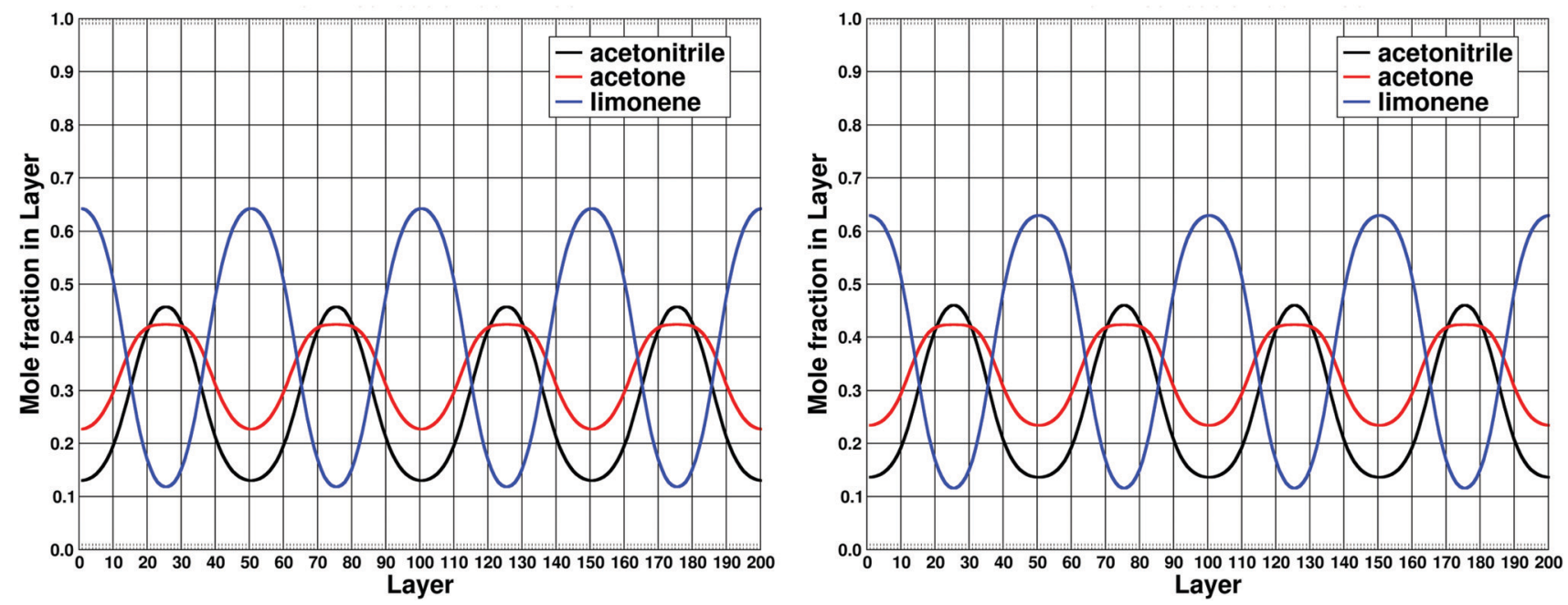

Fig. 4 COSMOplex results for acetonitrile/acetone/limonene at a phase composition of $0.30 / 0.35 / 0.35$ in mole fractions. Calculations performed on TZVPD-FINE level at a temperature of $25{ }^{\circ} \mathrm{C}$ with 8 s.o.s. phases and alternating polarity moments. Refined convergence thresholds were applied: 100 times lower (left-hand side) and 1000 times lower (right-hand side) than the COSMOplex default values. 
eventually vanish completely if the simulation is continued with stricter convergence criteria.

In contrast to that, relatively stable fluctuations could be obtained for the evaluated SFME and HBFME systems at phase compositions corresponding to points in the pre-Ouzo region of the ternary phase diagrams. This is shown on the example of acetonitrile/acetone/limonene (see Fig. 4) at an overall phase composition of $0.30 / 0.35 / 0.35$ in mole fractions, which is close to the LLE mixture critical point of the COSMO-RS-predicted phase diagram.

As already stated, COSMOplex can be used to evaluate the influence of the proximity to the LLE miscibility gap and to the LLE critical point in ternary phase diagrams on the extent of structuring in SFME and HBFME. As a proof of concept, we evaluated the results of COSMOplex calculations for the water/ ethanol/limonene mixture system, all at the same hydrotrope concentration but with three different water/oil ratios (see Fig. 5).

The phase composition of 0.05/0.70/0.25 and 0.25/0.70/0.05 in mole fractions, correspond to points located in the monophasic region of the COSMO-RS-predicted ternary phase diagram: one on the hydrotropic and the other on the lipotropic side of the LLE miscibility gap. The phase compositions of $0.15 / 0.70 / 0.15$ corresponds to a point within the LLE miscibility gap.

From the COSMOplex-predicted relative fluctuation amplitude heights and the corresponding path lengths of the fluctuations when plotted in the ternary phase diagram (see Fig. 5), we learn that the structuring in proximity of the LLE mixture critical point in the ternary phase diagram (lipotropic side) is significantly stronger when compared to a phase composition at the other side of the miscibility gap (hydrotropic side), but not as strong as at phase compositions within the miscibility gap (where the system will demix). Note that for overall phase compositions inside of the COSMO-RS-predicted miscibility gap: the upper and lower turning points of the COSMOplexpredicted fluctuations correspond to phase compositions close to the miscibility gap, which seem to be in good correlation with the COSMO-RS-predicted tie-lines in the ternary phase diagram (see Fig. 5). Hence, the chemical potentials of the compounds at the upper and lower turning points of the COSMOplex-predicted fluctuations, or alternatively, of the end-points of the corresponding fluctuation paths in the ternary phase diagram should be quite similar.

Instead of comparing the fluctuation amplitude heights or fluctuation path lengths corresponding to different points in the same ternary phase diagram (as shown e.g. in Fig. 5), COSMOplex can also be used to evaluate the effects of different hydrotropic compounds at fixed phase compositions. Within this contribution, the structuring of water/hydrotrope/anisole microemulsions with the hydrotropes ethanol, acetone and THF was investigated. A selection of results corresponding to various points with equimolar water/anisole ratio in the ternary phase diagrams of the SFME is composed in Fig. 6 and 7.
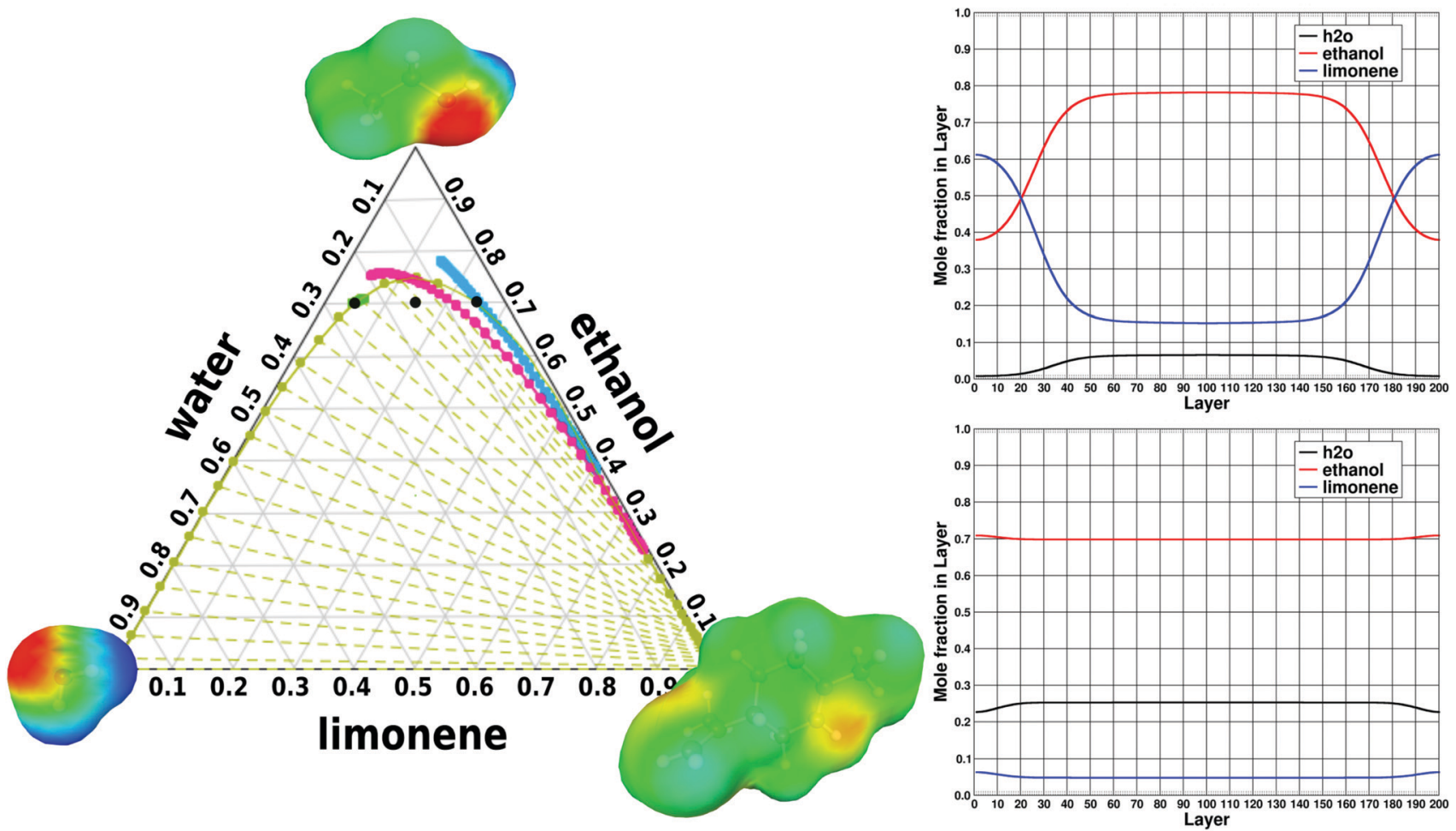

Fig. 5 Ternary phase diagram (flat green: predicted with COSMO-RS) of water/ethanol/limonene (left) - including COSMOplex-predicted fluctuations around the overall mole fraction phase compositions, which are indicated by the black dots in the ternary phase diagram - and COSMOplex results for the overall phase compositions of 0.05/0.70/0.25 (top right) and 0.25/0.70/0.05 (bottom right) in mole fractions. COSMO-RS and COSMOplex calculations were performed on TZVPD-FINE level at a temperature of $25^{\circ} \mathrm{C}$. 2 s.o.s. phases and alternating polarity moments $(\leftarrow \rightarrow)$ were used for the COSMOplex calculation set-up and convergence thresholds - being 100 times lower than the COSMOplex default values - were applied. 
Free energies of aggregation and self-organization for the COSMOplex-predicted fluctuations, are compiled in the ESI. $\dagger$ Please find additional COSMOplex results obtained for other phase compositions, calculation set-ups or convergence criteria, as well as plots of probability distribution curves and free energy curves compiled in the ESI. $\dagger$

For the water/ethanol/anisole system (see top row of Fig. 6 and left-hand side of Fig. 7), pronounced periodic fluctuations could be observed. The probability to find mesoscale inhomogeneities in the SFME increases with decreasing hydrotrope content and can be correlated with the relative heights of the COSMOplex-predicted fluctuation amplitudes or alternatively with the corresponding path lengths of the fluctuations when plotted in the ternary phase diagram.

Results indicate that the molecules in the SFME system are likely to form water-rich and anisole-rich compartments, which are stabilized by the ethanol molecules. The high affinity of the ethanol molecules to stabilize the interface of the formed compartments is e.g. indicated by the location of the maximum turning points in the ethanol layer composition curves (see Fig. 6), which are closely related to probability distribution curves (see plots compiled in the ESI $\dagger$ ) indicating the probability to find a molecule in a given COSMOplex simulation box layer. These findings are in perfect agreement with literature. ${ }^{4,5,11,36}$

In case of the water/acetone/anisole and water/THF/anisole mixture systems (see middle and bottom row of Fig. 6 and right-hand side of Fig. 7), only ill-defined fluctuations were obtained, indicating the presence of relatively homogeneous and "bulk-like" compartments and water-rich aggregates. Note that the number and position of the peaks depend on the reasonable definition of the simulation box and the corresponding calculation set-up (see ESI $\dagger$ ). Although acetone and THF show the tendency to aggregate at the interfaces between the water-rich aggregates and the "bulk-like" compartments,
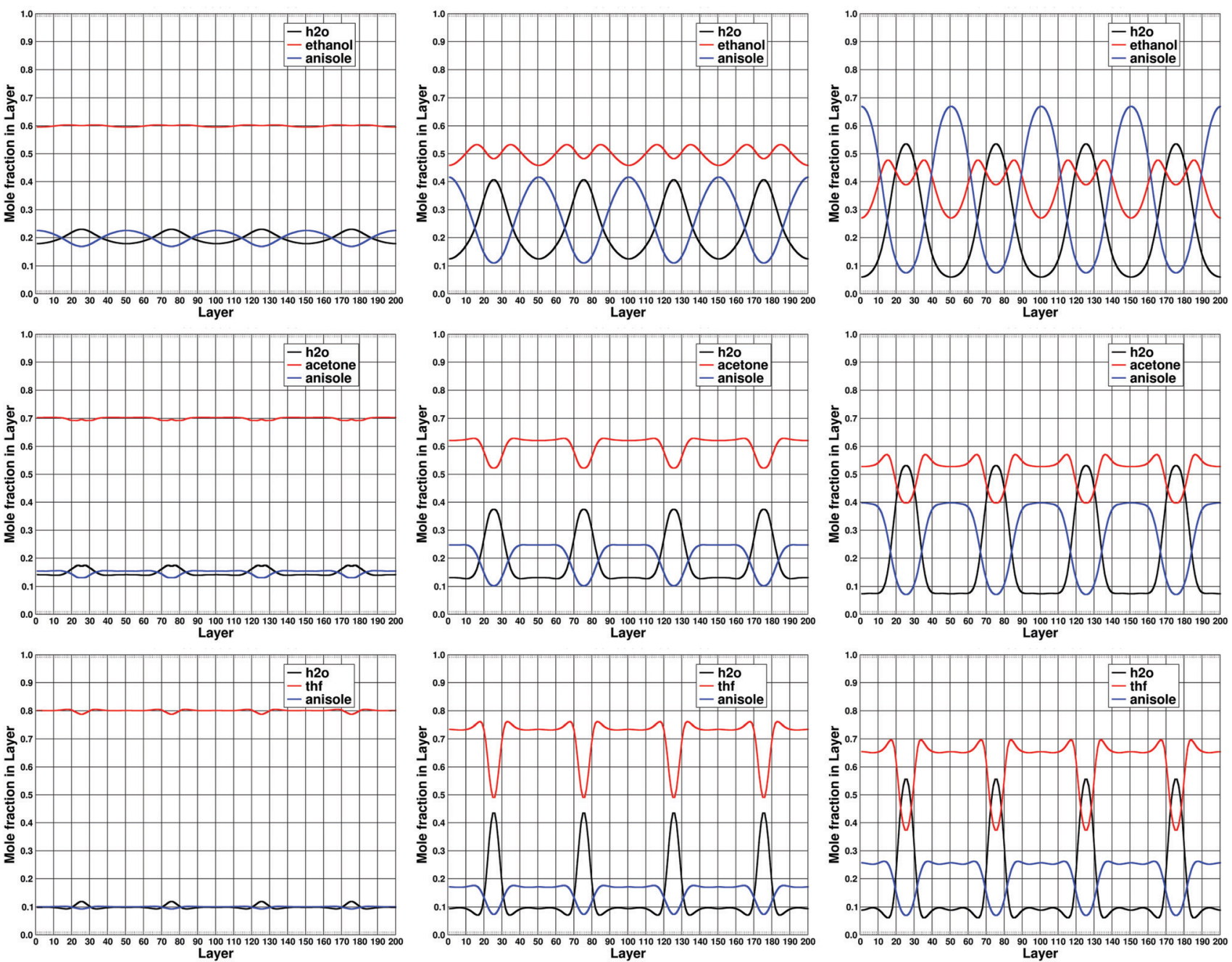

Fig. 6 COSMOplex results for water/ethanol/anisole (top row), water/acetone/anisole (middle row) and water/THF/anisole (bottom row) at given phase compositions in mole fractions. Water/ethanol/anisole: 0.20/0.60/0.20 (left), 0.25/0.50/0.25 (middle) and 0.30/0.40/0.30 (right). Water/acetone/anisole: 0.15/0.70/0.15 (left), 0.20/0.60/0.20 (middle) and 0.25/0.50/0.25 (right). Water/THF/anisole: 0.10/0.80/0.10 (left), 0.15/0.70/0.15 (middle) and 0.20/0.60/ 0.20 (right). Calculations performed on TZVPD-FINE level at a temperature of $25{ }^{\circ} \mathrm{C}$ with 8 s.o.s. phases and alternating polarity moments. Refined convergence thresholds - being 100 times lower than the COSMOplex default values - were applied. 

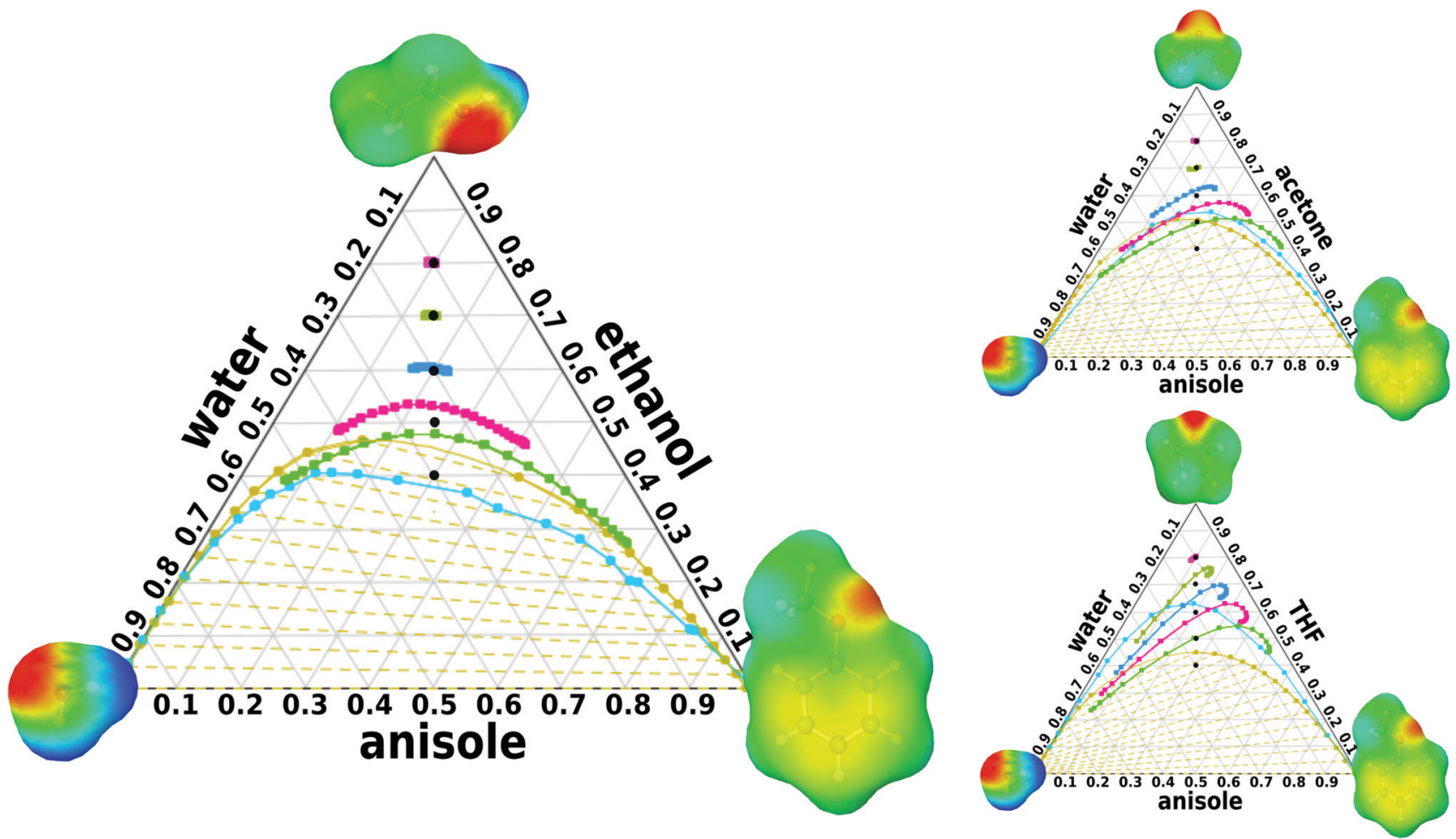

Fig. 7 Ternary phase diagrams (light blue: experiment, flat green: predicted with COSMO-RS) of water/ethanol/anisole (left), water/acetone/anisole (top right) and water/THF/anisole (bottom right) - including COSMOplex-predicted fluctuations around the overall mole fraction phase compositions, which are indicated by the black dots in the ternary phase diagrams. COSMO-RS and COSMOplex calculations were performed on TZVPD-FINE level at a temperature of $25^{\circ} \mathrm{C} .8$ s.o.s. phases and alternating polarity moments were used for the COSMOplex calculation set-up and convergence thresholds being 100 times lower than the COSMOplex default values - were applied.

no defined mesoscale structuring can be expected from the COSMOplex results for the water/hydrotrope/anisole systems with acetone or THF acting as the hydrotropic component.

These findings are in perfect agreement with the experimental DLS results (compiled in Fig. 8), measured at phase compositions slightly above the LLE miscibility gap. Note that significant correlation functions were only observed for the ternary water/ethanol/anisole SFME system. Binary mixtures of water/ethanol do not show any correlation functions, indicating a molecularly distributed binary solvent mixture as forwarded recently. ${ }^{37}$
Upon the addition of $(5,10,15,20$ and $25 \mathrm{~mol} \%)$ anisole to the water/ethanol mixture, a gradual formation of well-defined correlation functions with an increasing shift to higher decay times was observed. DLS measurements on binary water/THF mixtures already show mesoscale compartmentation. This pre-structuring gets more and more destroyed upon the addition of a third hydrophobic component, as forwarded recently, resulting in less pronounced correlation functions. ${ }^{37}$

For the water/acetone/anisole and water/THF/anisole systems, only weak and unresolved signals - but no mesoscale structuring could be observed. However, the detected signals might correspond
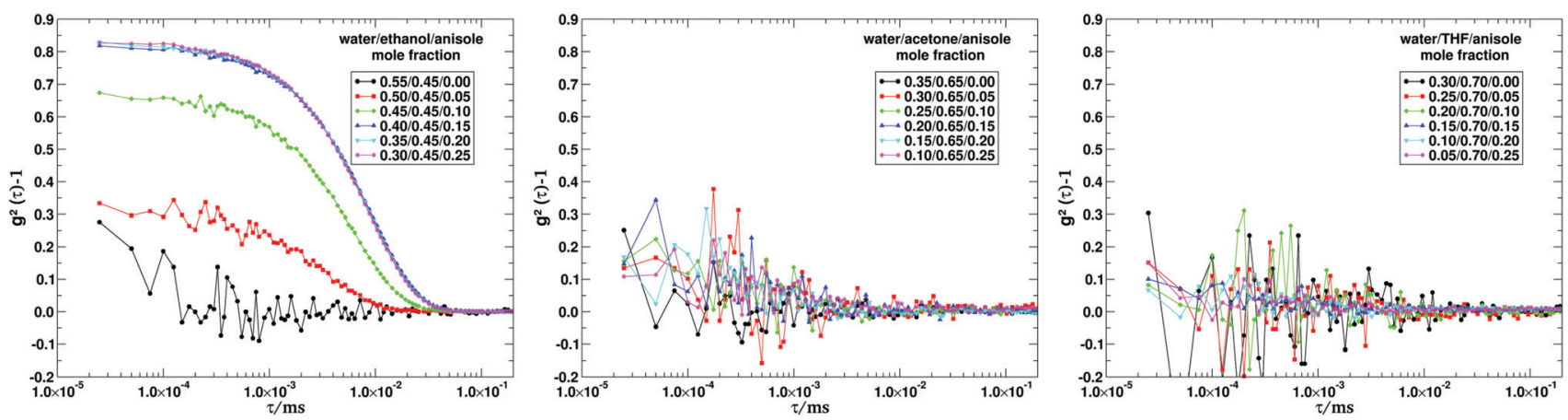

Fig. 8 Normalized intensity autocorrelation functions $g^{(2)}(\tau)-1$ obtained by DLS measurements for ternary mixtures: water/ethanol/anisole (left), water/ acetone/anisole (middle) and water/THF/anisole (right) at $25{ }^{\circ} \mathrm{C}$. 
to fast-fluctuating water-rich aggregates. Be aware that due to the lower miscibility gap in the water/acetone/anisole system, the corresponding DLS autocorrelation function were measured at a phase composition with lower hydrotrope content and hence larger water-rich aggregates can be expected.

\section{Correlation between mesoscale structuring of SFME and HBFME and the hydrotropic efficiencies}

Although the COSMO-RS-predicted ternary phase diagrams for water/ethanol/anisole and water/acetone/anisole are in good agreement with the experimental results (see phase diagrams in Fig. 7), larger deviations were observed for the water/THF/ anisole system. Hence, the trends for the hydrotropic efficiencies from the experimental phase diagrams - ethanol > acetone $>$ THF - could not be reproduced completely. However, the deviations from the experiment may to a large extent arise from the fact that COSMO-RS assumes the liquid mixture to be homogeneous and furthermore does not take into account correlation effects of interacting molecules.

Nevertheless, COSMO-RS predictions and $\sigma$-profiles can provide valuable information on the molecular interactions, as we describe in the ESI. $\dagger$ The hydrotropic efficiencies of ethanol, acetone and THF in water/hydrotrope/oil systems can - as already observed by Bauduin et al. ${ }^{8}$ - be correlated with the affinity of the hydrotropic compound towards the water-rich phase in infinite dilution and the amount of non-polar molecular surface of the corresponding hydrotrope. The first can be quantified either by infinite dilution activity coefficients or the partition coefficients of hydrotrope molecules in infinite dilution between the immiscible phases in a binary water-oil system in equilibrium, the second can be obtained from the $\sigma$-profiles of the hydrotrope molecules.

The COSMOplex results presented in this study show another interesting connection between mesoscale structuring in SFME and HBFME and the hydrotropic efficiencies. We found that the amplitude heights of fluctuations in the free energies of aggregation and self-organization as well as the relative amplitude heights of COSMOplex-predicted fluctuations and the corresponding path lengths of the fluctuations in the ternary phase diagram are strongly dependent on the overall phase composition of the ternary mixtures. Furthermore, results indicate that the most pronounced structuring is likely to occur in the monophasic region of the ternary phase diagram in proximity to the miscibility gap or, more precisely, to the LLE critical point.

Hence, the question arises if structuring in the pre-Ouzo region (and hence in proximity to the LLE mixture critical point) of a ternary phase diagram has its origin in critical fluctuations. However, this assumption was already disproved by Zemb et al. ${ }^{4}$ COSMOplex results for the evaluated water/ hydrotrope/anisole mixture systems indicate that the realms of miscibility and the location of the LLE miscibility gap in ternary phase diagrams might be strongly linked to structuring and aggregation of the molecules in SFME and HBFME systems. In fact, aggregate structures and compartments, getting too large to be stabilized by hydrotrope or surfactant molecules, are a reasonable explanation for the occurrence of a LLE miscibility gap in ternary phase diagrams.

In case of the evaluated water/ethanol/anisole system, we have seen that a reduction of the ethanol content increases the fluctuation path lengths in the ternary phase diagram - as shown on the left-hand side of Fig. 7 - and results in more pronounced structuring of the SFME system into water-rich and anisole-rich compartments, with ethanol molecules being preferably at the compartment interfaces (see top row of Fig. 6). This is in agreement with ideas forwarded by the group of Horinek et al. ${ }^{36}$ and the group of Zemb et al. ${ }^{4,7}$ However, below a system and temperature dependent threshold, the microemulsion can no longer be stabilized by the reduced number of ethanol molecules and the system will separate in a hydrophilic and a hydrophobic phase. The pronounced structuring in the pre-Ouzo region of the SFME could also be observed with the (bulk $\leftarrow \rightarrow \leftarrow \rightarrow \leftarrow \rightarrow$ bulk) calculation set-up and periodic boundary conditions (see Fig. 9, left).

Mean compound free energies of aggregation and selforganization in the evaluated water/hydrotrope/anisole systems (compiled in the ESI $\dagger$ ) indicate that the aggregation of hydrotrope and anisole molecules can be seen as the driving force for the observed microemulsion-like structuring in the monophasic region of the SFME. At overall phase compositions corresponding to points within the miscibility gap, the stabilizing effect of the
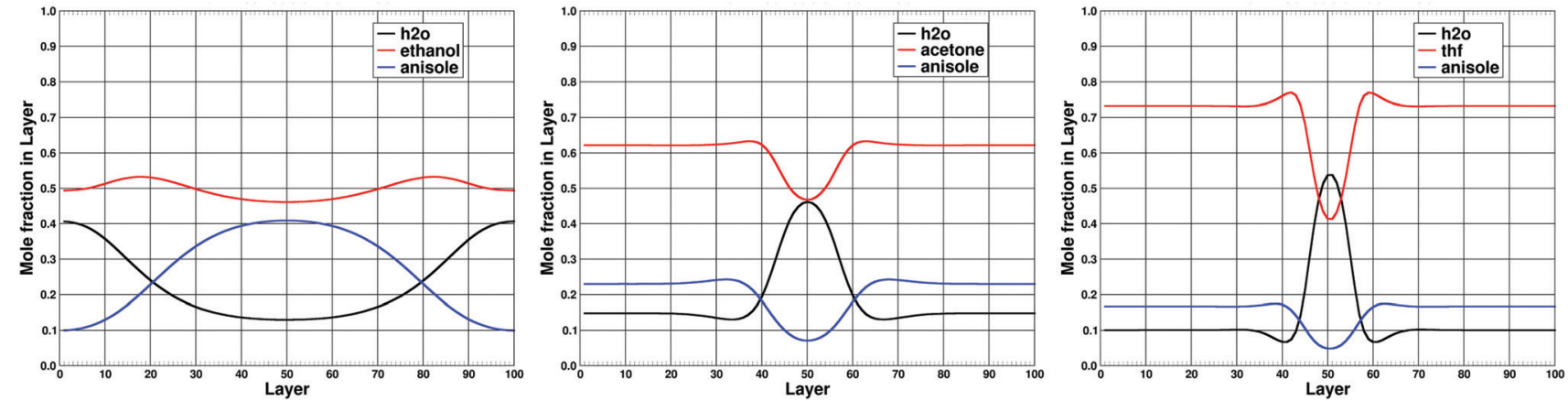

Fig. 9 COSMOplex results for water/ethanol/anisole (left, phase composition: 0.25/0.50/0.25), water/acetone/anisole (middle, phase composition: 0.20/0.60/0.20) and water/THF/anisole (right, phase composition: 0.15/0.70/0.15). Calculations performed on TZVPD-FINE level at a temperature of $25{ }^{\circ} \mathrm{C}$ with (bulk $\leftarrow \rightarrow \leftarrow \rightarrow \leftarrow$ bulk) calculation set-up. Refined convergence thresholds - being 1000 times lower than the COSMOplex default values - were applied. 

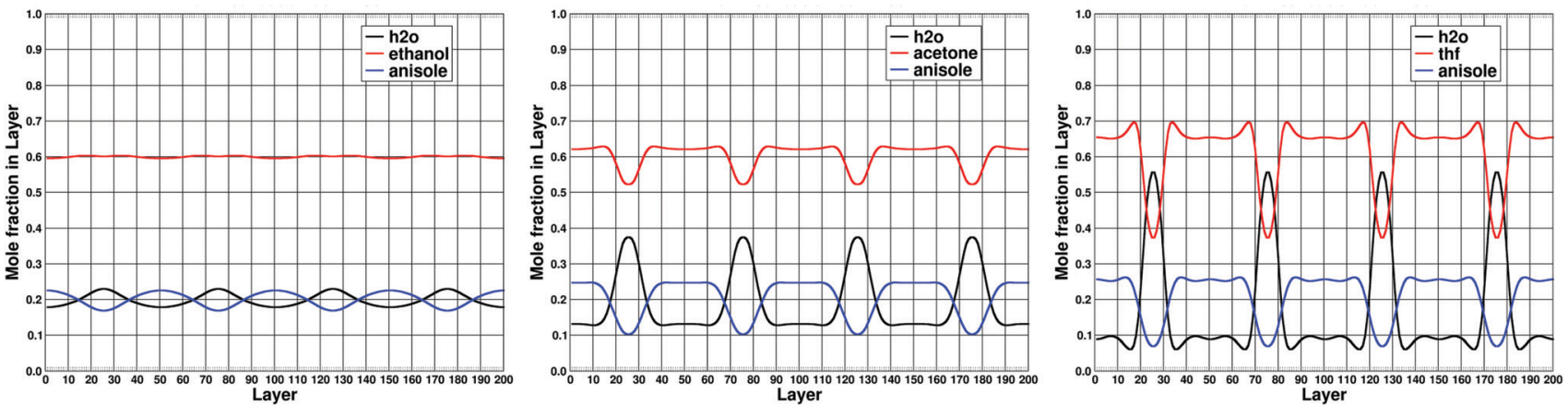

Fig. 10 COSMOplex results for water/ethanol/anisole (left), water/acetone/anisole (middle) and water/THF/anisole (right) at a phase composition of 0.20/0.60/0.20 in mole fractions. Calculations performed on TZVPD-FINE level at a temperature of $25^{\circ} \mathrm{C}$ with 8 s.o.s. phases and alternating polarity moments. Refined convergence thresholds - being 100 times lower than the COSMOplex default values - were applied.

hydrotrope molecules is lost and the self-aggregation of the immiscible components water and anisole is the driving force for aggregation or, more precisely, for the phase separation.

In case of the water/acetone/anisole system and water/THF/ anisole system, no periodic fluctuation in the layer compositions could be observed in simulations started with the (bulk $\leftarrow$ $\rightarrow \leftarrow \rightarrow \leftarrow \rightarrow$ bulk) calculation set-up (see Fig. 9). Instead, the COSMOplex results indicate the formation of water-rich aggregates and relatively homogeneous, "bulk-like" compartments in the pre-Ouzo regions of the corresponding ternary phase diagrams (see right-hand side of Fig. 7). The first is displayed e.g. by peaks in the water curves, the second by relatively constant layer compositions in the residual COSMOplex simulation box layers. A reduction of the hydrotrope (acetone or THF) content in these systems leads to larger and more pronounced aggregates with higher water content. Note that the higher numbers of peaks corresponding to water-rich aggregates in the COSMOplex results shown in Fig. 6 have their origin in a different calculation set-up and are not a sign of periodic fluctuations. Nevertheless, the acetone and THF molecules still serve as solubilizers for the immiscible components and the system will demix if the hydrotrope content gets too low.

Summarizing, the hydrotropic efficiency evaluates how well the aggregates and compartments can be stabilized by the hydrotrope molecules in order to prevent the system from demixing. Due to the fact that mixture systems are assumed to be homogeneous within the COSMO-RS calculation, the observed trend for the hydrotropic efficiencies as indicated by the experimental phase diagrams could not be reproduced by use of COSMO-RS-predicted phase diagrams. However, by use of the COSMOplex model, it is possible to take mesoscale inhomogeneities into account and evaluate the influence of different hydrotropes at a given phase composition.

Further, the heights of COSMOplex-predicted fluctuation amplitudes (or peaks corresponding to water-rich aggregates) at the same phase composition but with different hydrotropes in SFME systems - or alternatively, the corresponding fluctuation path lengths in the ternary phase diagrams - can be used as a measure for the hydrotropic efficiencies. However, the chosen overall phase composition should correspond to a point located within the monophasic region in all considered ternary phase diagrams. In this case, for a fixed phase composition, the less pronounced structuring, which is indicated by the shorter fluctuation path length in the ternary phase diagram, corresponds to the hydrotropic compound with the higher hydrotropic efficiency.

From the COSMOplex results obtained for the water/ hydrotrope/anisole system at mole fractions of $0.20 / 0.60 / 0.20$ for the hydrotropes ethanol, acetone and THF (shown in Fig. 7 and in Fig. 10), the following order was found for the hydrotropic efficiencies: ethanol $>$ acetone $>$ THF. However, pronounced mesoscale structuring was only observed for the water/ethanol/ anisole system.

\section{Conclusion}

The experimentally investigated ternary mixtures have been simulated by the COSMOplex method. In these simulations, distinct composition fluctuations were found in those systems, in which the experiment shows mesoscopic structuring of the liquids and it thus seems that COSMOplex can predict the appearance of mesoscopic structuring in ternary mixtures and, in addition, helps to analyze and further understand the compartmentalization phenomena of SFME and HBFME.

Within this contribution, all COSMOplex calculations are based on layered simulation boxes with periodic boundary conditions and equally distributed molecules. Thus, at a defined overall phase composition, all initial phase compositions of bulk and self-organizing system (s.o.s.) phases are exactly equal and the phases differ only in the orientation and the considered conformations of the molecules in the COSMOplex simulation box. This allows the assignment of COSMOplex results, i.e. of the predicted fluctuations in the phase composition and the free energies, to the corresponding points in the ternary phase diagrams and enables to evaluate in which regions of the ternary phase diagram mesoscopic structuring is rather likely.

Interesting insights into the rather complex topic of selforganizing-systems and SFME formation could be gained from the results of the performed COSMOplex calculations and verified by experimental phase diagrams and DLS measurements. 
For instance, we were able to show that mesoscale structuring and hydrotropic efficiencies correlate: the hydrotrope with the higher hydrotropic efficiency displays weaker mesoscale structuring at the same phase composition as the hydrotrope with the lower hydrotropic efficiency. Furthermore, our results indicate that the occurrence and the location of an LLE miscibility gap in a ternary phase diagram is in strong relation with aggregation and compartmentation - and therefore also depends on the hydrotropic, lipotropic and co-solvency efficiencies of the hydrotropic compound in a SFME or HBFME.

We found pronounced microemulsion-like structuring in ternary systems of molecules without any hydrogen-bond donor groups, and hence, without hydrogen-bonding. This is an extraordinary finding, which was not reported in literature, to the best of our knowledge. For the presented acetonitrile/ acetone/limonene system and other systems of that kind, we introduced the term hydrogen-bonding-free microemulsions (HBFME) as a sub-group of SFME. In fact, from COSMOplex results and experimental DLS measurements, we learned that hydrogen-bonding re-enforces microemulsion-like structuring, but is not a prerequisite for it.

Summarizing, the COSMOplex method clearly extends the predictive abilities of COSMO-RS towards inhomogeneous, structured liquid systems like SFME and HBFME and might become a valuable supplement to Molecular Dynamics simulations for the computer-based analysis of self-organizing systems. However, it needs to be emphasized that the applied version is a prototype and that the free energy differences in the formation of aggregate structures in the investigated systems are in the range of $0.05 \mathrm{kcal} \mathrm{mol}^{-1}$ and thus close to the expected accuracy of COSMOplex. As a consequence, no absolute values of properties (like e.g. layer compositions or free energies) have been interpreted in this contribution. Instead we compared COSMOplex-predicted fluctuations or, more precisely, the corresponding fluctuation path lengths or relative amplitude heights - as a measure for the propensity of the molecules to find mesoscale inhomogeneities in SFME and HBFME - at different phase compositions in ternary phase diagrams.

\section{Conflicts of interest}

There are no conflicts to declare.

\section{Acknowledgements}

We thank Thomas Zemb and Johannes Schwöbel for fruitful discussions about SFME and the COSMOplex method. M. Hahn thankfully acknowledges the financial and scientific support of Andreas Klamt, COSMOlogic GmbH \& Co. KG, and Werner Kunz, Institute of Physical and Theoretical Chemistry, University of Regensburg. S. Krickl thanks the Fonds der chemischen Industrie (FCI) for his scholarship (grant number 197181).

\section{Notes and references}

1 M. L. Klossek, D. Touraud, T. Zemb and W. Kunz, ChemPhysChem, 2012, 13, 4116-4119.

2 V. Fischer, J. Marcus, D. Touraud, O. Diat and W. Kunz, J. Colloid Interface Sci., 2015, 453, 186-193.

3 W. Kunz, K. Holmberg and T. Zemb, Curr. Opin. Colloid Interface Sci., 2016, 22, 99-107.

4 T. N. Zemb, M. Klossek, T. Lopian, J. Marcus, S. Schöettl, D. Horinek, S. F. Prevost, D. Touraud, O. Diat, S. Marčelja and W. Kunz, Proc. Natl. Acad. Sci. U. S. A., 2016, 113, 4260-4265.

5 S. Schöttl, J. Marcus, O. Diat, D. Touraud, W. Kunz, T. Zemb and D. Horinek, Chem. Sci., 2014, 5, 2949-2954.

6 P. Bošković, V. Sokol, T. Zemb, D. Touraud and W. Kunz, J. Phys. Chem. B, 2015, 119, 9933-9939.

7 T. Lopian, S. Schöttl, S. Prévost, S. Pellet-Rostaing, D. Horinek, W. Kunz and T. Zemb, ACS Cent. Sci., 2016, 2, 467-475.

8 P. Bauduin, A. Renoncourt, A. Kopf, D. Touraud and W. Kunz, Langmuir, 2005, 21, 6769-6775.

9 P. Bauduin, F. Testard and T. Zemb, J. Phys. Chem. B, 2008, 112, 12354-12360.

10 S. H. Yalkowsky, Solubility and solubilization in aqueous media, American Chemical Society, Oxford University Press, Washington, D.C.: New York, 1999.

11 O. Diat, M. L. Klossek, D. Touraud, B. Deme, I. Grillo, W. Kunz and T. Zemb, J. Appl. Crystallogr., 2013, 46, 1665-1669.

12 M. L. Klossek, D. Touraud and W. Kunz, Phys. Chem. Chem. Phys., 2013, 15, 10971-10977.

13 Y. L. Khmelnitski, R. Hilhorst and C. Verger, Eur. J. Biochem., 1988, 176, 265-271.

14 S. Krickl, D. Touraud, P. Bauduin, T. Zinn and W. Kunz, J. Colloid Interface Sci., 2018, 516, 466-475.

15 S. Krickl, T. Buchecker, A. U. Meyer, I. Grillo, D. Touraud, P. Bauduin, B. König, A. Pfitzner and W. Kunz, Phys. Chem. Chem. Phys., 2017, 19, 23773-23780.

16 S. Krickl, L. Jurko, K. Wolos, D. Touraud and W. Kunz, J. Mol. Liq., 2018, 271, 112-117.

17 J. Marcus, M. L. Klossek, D. Touraud and W. Kunz, Flavour Fragrence J., 2013, 28, 294-299.

18 U. Mohorič, A. Beutner, S. Krickl, D. Touraud, W. Kunz and F.-M. Matysik, Anal. Bioanal. Chem., 2016, 408, 8681-8689.

19 S. Abbott, J. J. Booth and S. Shimizu, Green Chem., 2017, 19, 68-75.

20 S. Shimizu and Y. N. Kanasaki, J. Mol. Liq., 2019, 274, 209-214.

21 S. Shimizu and N. Matubayasi, Phys. Chem. Chem. Phys., 2017, 19, 23597-23605.

22 S. Shimizu and N. Matubayasi, Phys. Chem. Chem. Phys., 2016, 18, 25621-25628.

23 S. Shimizu, J. Booth and S. Abbott, Phys. Chem. Chem. Phys., 2013, 15, 20625-20632.

24 A. Klamt, J. Phys. Chem., 1995, 99, 2224.

25 A. Klamt, V. Jonas, T. Bürger and J. C. W. Lohrenz, J. Phys. Chem. A, 1998, 102, 5074. 
26 A. Klamt, COSMO-RS From Quantum Chemistry to Fluid Phase Thermodynamics and Drug Design, Elsevier, 2005.

27 A. Klamt, L. Koch, S. Terzi, U. Huniar, J. Schwöbel and T. Gaudin, ChemRxiv, 2018, DOI: 10.26434/chemrxiv. 7218245.v1.

28 F. Eckert and A. Klamt, COSMOtherm, Version C3.0, Release 15.01, COSMOlogic GmbH \& Co. KG, Imbacher Weg 46, D-51379 Leverkusen, Germany, 2014.

29 COSMOtherm, Release 18, 2018.

30 A. D. Becke, Phys. Rev. A: At., Mol., Opt. Phys., 1988, 38, 3098-3100.

31 J. P. Perdew, Phys. Rev. B: Condens. Matter Mater. Phys., 1986, 33, 8822-8824.
32 A. Klamt and G. Schüürmann, J. Chem. Soc., Perkin Trans. 2, 1993, 799.

33 A. Klamt and F. Eckert, Fluid Phase Equilib., 2000, 172, 43-72.

34 T. Gaudin, I. Pezron and A. Klamt, J. Surfactants Deterg., 2018, DOI: $10.1002 /$ jsde.12225.

35 M. L. Dekker, M. Clausse, H. L. Rosano and A. S. Zradba, Microemulsion Systems, Surfactant Science Ser., New York, PA, 24th edn, 1987.

36 S. Schöttl, D. Touraud, W. Kunz, T. Zemb and D. Horinek, Colloids Surf., A, 2015, 480, 222-227.

37 T. Buchecker, S. Krickl, R. Winkler, I. Grillo, P. Bauduin, D. Touraud, A. Pfitzner and W. Kunz, Phys. Chem. Chem. Phys., 2017, 19, 1806-1816. 